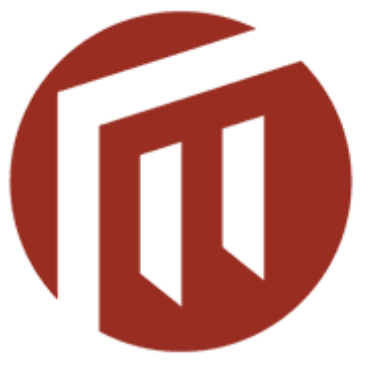

MALMÖ UNIVERSITY
Malmö University Electronic Publishing

This is an author produced version of a paper published in 2008 19th International Conference on Pattern

Recognition. This paper has been peer-reviewed but does not include the final publisher proof-corrections or journal pagination.

Citation for the published paper:

Mattias Hansson, Niels-Christian Overgaard, Anders Heyden, "Rayleigh segmentation of the endocardium in ultrasound images", 2008: pp 1-4.

URL: 10.1109/ICPR.2008.4761334

Access to the published version may require subscription.

Published with permission from: IEEE 


\title{
Rayleigh Segmentation of the Endocardium in Ultrasound Images*
}

\author{
Mattias Hansson, Niels Chr. Overgaard and Anders Heyden \\ Applied Mathematics Group, Malmö University, Sweden \\ \{mattias.hansson,nco,heyden\}@mah.se
}

\begin{abstract}
In this paper we present the Coupled Active Contours (CAC) method, which is applied to segmentation of the endocardium in ultrasonic images assuming Rayleigh distributed intensities. Comparative experiments, both real and synthetic, with a standard prior model are presented. In the CAC model the prior acts, by affine transformation, on the same image information as the active contour, in addition to the traditional interaction between prior and active contour. By this higher convergence rate and robustness, w.r.t artifacts and poor initialization, is achieved.
\end{abstract}

\section{Introduction}

Ultrasound is a low-cost, widely available diagnostic tool, and as such much is to be gained by improving its efficiency. Unfortunately the analysis of ultrasound images is often hindered by so-called speckle contamination. Speckle arises when the ultrasound beam is reflected from different parts of the tissue. The Rayleigh distribution has been suggested for modeling the ultrasonic $B$-scan in Sarti et al. [10], and it is also used in the model employed in this paper.

The vast majority of methods developed for ultrasound segmentation incorporate prior information. In Qian et al. [7] a complex segmentation model involving a prior model for signal dropouts. The log-normal distribution was used for the image models. Also a novel search technique called "tunneling descent" was used to avoid trapping in local minima. Chen et al. [2] combines an edge-based active contour with shape priors to obtain segmentations of ultrasound and MRI images. No statistical image models are involved. In [1] an intensity prior was added to the model.

The work most closely related to the CAC model is Dydenko et al. [4], where a two-step method is pro-

\footnotetext{
* Thanks to Dr. Petri Gudmundsson (Dept. of Cardiology, Malmö University Hospital, Sweden) for providing clinical advice.
}

posed which combines an initial affine "alignment" of the previous segmentation result to the current frame, followed by a Rayleigh segmentation where the aligned contour is used as initialization. Only local rigid transformations are used in contrast to the CAC model which allows global affine "alignment" of the prior, to guide the active contour over greater distances towards the desired object. For more examples of incorporating shape priors in segmentation see [3, 8, 9, 5, 2].

In this paper we apply the Coupled Active Contours model (CAC) to a set of images, both synthetic and real. The CAC model is defined as follows:

$$
\begin{array}{r}
\Gamma^{*}=\underset{\Gamma, T}{\arg \min }\left\{E_{\mathrm{CAC}}\left(\Gamma, T \Gamma_{0}\right):=\alpha E_{\text {Ray }}(\Gamma)+\right. \\
\left.\beta E_{\text {Ray }}\left(T \Gamma_{0}\right)+\gamma E_{\mathrm{I}}\left(\Gamma, T \Gamma_{0}\right)\right\},
\end{array}
$$

where $\Gamma$ denotes the active contour, $\Gamma_{0}$ a prior contour and $\alpha, \beta, \gamma>0$ are weight parameters. $T$ ranges over a group of transformations, so that the interaction becomes pose invariant. Here $T \Gamma_{0}$ denotes the transformed contour, and $\gamma$ is a coupling constant which determines the strength of the contour interaction $E_{\mathrm{I}}$. $E_{\text {Ray }}$ denotes a Rayleigh functional, see Section 2. In this way we obtain a model in which the active- and prior contours are treated on an (almost) equal footing, hence we speak of Coupled Active Contours. We refer to $T \Gamma_{0}$ as the sensitive prior, because it interacts with the image. The latter may be a shape prior, or a socalled deformable shape prior, as in our case, cf. Section 2.

The idea is to let the sensitive prior guide the active contour towards the desired object, resulting in a more robust and rapidly converging segmentation. The CAC model is compared to a standard prior-based model (SPS),

$$
\Gamma^{*}=\underset{\Gamma, T}{\arg \min }\left\{E_{\text {Ray }}(\Gamma)+\gamma E_{\mathrm{I}}^{\triangle}\left(\Gamma, T \Gamma_{0}\right)\right\},
$$

with interaction as in [3], cf. Eq. (9) Section 2, and allowing affine transformation of the prior. 


\section{The Segmentation Model}

Rayleigh Segmentation. Our main impetus for using the Rayleigh distribution in modeling the ultrasonic $B$-scan images comes from Sarti et al. [10]. In the continuous setting, a gray scale image is considered to be a real valued function $I: D \rightarrow[0, \infty)$ defined on the image domain $D \subset \mathbf{R}^{2}$. A point $\mathbf{x} \in D$ is referred to as a pixel, $I(\mathbf{x})$ as the corresponding gray scale value. The interior/exterior of $(\cdot)$ is denoted by int $(\cdot) / \operatorname{ext}(\cdot)$ and the area of $(\cdot)$ by $|\cdot|$. Rayleigh segmentation is an $a c$ tive contour model where the idea is to find a contour $\Gamma$, such that the image $I$ is optimally approximated by the following statistical image model:

$$
I(\mathbf{x}) \sim\left\{\begin{array}{ll}
\operatorname{Ray}\left(\sigma_{\text {int }}^{2}\right) & \text { if } \mathbf{x} \in \operatorname{int}(\Gamma) \\
\operatorname{Ray}\left(\sigma_{\text {ext }}^{2}\right) & \text { if } \mathbf{x} \in \operatorname{ext}(\Gamma)
\end{array} .\right.
$$

Here $X \sim \operatorname{Ray}\left(\sigma^{2}\right)$ means that the random variable $X$ is Rayleigh distributed with parameter $\sigma^{2}$, i.e. has the probability density function (pdf) $f_{X}(x)=$ $\left(x / \sigma^{2}\right) \exp \left(-x^{2} / 2 \sigma^{2}\right), x \geq 0$ and $f_{X}(x)=0$ otherwise. The Rayleigh functional is the given by,

$$
\begin{aligned}
E_{\text {Ray }}(\Gamma)=\nu|\Gamma| & +|\operatorname{int}(\Gamma)| \log \left(\sigma_{\text {int }}^{2}(\Gamma)\right)+ \\
& +|\operatorname{ext}(\Gamma)| \log \left(\sigma_{\text {ext }}^{2}(\Gamma)\right),
\end{aligned}
$$

where $\nu>0$ is a regularization parameter,

$$
\sigma_{\text {int }}^{2}=\frac{1}{|\operatorname{int}(\Gamma)|} \int_{\operatorname{int}(\Gamma)} \frac{I(\mathbf{x})^{2}}{2} d \mathbf{x}
$$

and $\sigma_{\text {ext }}^{2}$ is computed similarly. Let the contour $\Gamma$ be represented as the zero-level set of the time dependent function function $\phi=\phi(t, \mathbf{x})$ as $\Gamma=\{\mathbf{x} \in$ $\left.\mathbf{R}^{2} ; \phi(t, \mathbf{x})=0\right\}$ with $\operatorname{int}(\Gamma)=\{\mathbf{x} ; \phi(\mathbf{x})<0\}$, cf. [6]. Then the contour is evolved in time towards a (local) minimum by solving the level set PDE,

$$
\frac{\partial \phi}{\partial t}=\left[\nu \kappa+\log \left(\frac{\sigma_{\text {int }}^{2}}{\sigma_{\text {ext }}^{2}}\right)+I(\mathbf{x})^{2}\left(\frac{1}{\sigma_{\text {int }}^{2}}-\frac{1}{\sigma_{\text {ext }}^{2}}\right)\right]|\nabla \phi|,
$$

Interaction: The Deformable Shape Prior. The affine pose-invariant interaction $E_{\mathrm{I}}$ between two contours $\Gamma$ and $\Gamma_{0}$ is defined by the integral

$$
E_{\mathrm{I}}\left(\Gamma, T \Gamma_{0}\right)=\left|\operatorname{det} T^{-1}\right| \int_{\operatorname{int}(\Gamma)}\left(\frac{\phi_{0}\left(T^{-1} \mathbf{x}\right)}{W}\right)^{3} d \mathbf{x} .
$$

where $\phi_{0}=\phi_{0}(\mathbf{y})$ denotes the signed distance function for $\Gamma_{0}$, and the parameter $W>0$ defines the reach of the interaction. The associated $L^{2}$-gradient is:

$$
\nabla_{\Gamma} E_{I}\left(\Gamma, T \Gamma_{0}\right)=\left|\operatorname{det} T^{-1}\right|\left(\frac{\phi_{0}\left(T^{-1} \mathbf{x}\right)}{W}\right)^{3} .
$$

For points close to the sensitive prior, the $L^{2}$-gradient is small. Therefore the interaction between nearby contours will be weak. This is desirable because it allows the active contour to adapt to the image information in a neighbourhood of width $\approx W$ around the prior.

Interaction: Area of the Set Symmetric Difference. We compare the proposed method to results obtained using the standard prior-based method (2) with the interaction given by the symmetric set difference as in e.g. [3]:

$$
\begin{gathered}
E_{\mathrm{I}}^{\triangle}\left(\Gamma, \Gamma_{0}\right)=\operatorname{area}\left(\operatorname{int}(\Gamma) \triangle \operatorname{int}\left(\Gamma_{0}\right)\right)= \\
=\int\left|H(-\phi(\mathbf{x}))-H\left(-\phi_{0}(\mathbf{x})\right)\right|^{2} d \mathbf{x},
\end{gathered}
$$

where $H$ is the usual Heaviside function. The $L^{2}$ gradient is $\nabla_{\Gamma} E_{\mathrm{I}}^{\triangle}(\Gamma)=1-2 H\left(-\phi_{0}(\mathbf{x})\right)$. We refer to segmentation using the pose-invariant functional in (2) with $E_{\mathrm{I}}=E_{\mathrm{I}}^{\triangle}$ as Standard Prior segmentation (SPS).

The Affine Group. Orientation preserving affine maps $T$ play an important role in the paper. They form a group under composition which may be parametrized by six parameters, $\boldsymbol{\rho}=\left(\mu, \theta, s_{x}, s_{y}, a_{x}, a_{y}\right) \in \mathbf{R}_{+} \times$ $[-\pi, \pi] \times \mathbf{R}^{4}$, where $\mathbf{a}=\left(a_{x}, a_{y}\right)$ and

$$
A=\mu\left(\begin{array}{cc}
\cos \theta & \sin \theta \\
-\sin \theta & \cos \theta
\end{array}\right)\left(\begin{array}{cc}
1 & s_{x} \\
0 & 1
\end{array}\right)\left(\begin{array}{cc}
1 & 0 \\
s_{y} & 1
\end{array}\right) .
$$

We use $\mathbf{y}=T(\boldsymbol{\rho}) \mathbf{x}$ as a shorthand for the corresponding affine transformation.

The CAC Gradient Descent Flow. The minimization problem (1) becomes $\left(\Gamma^{*}, \rho^{*}\right)=$ $\arg \min _{\Gamma, \boldsymbol{\rho}} E_{\mathrm{CAC}}\left(\Gamma, T(\boldsymbol{\rho}) \Gamma_{0}\right)$, which is solved using a standard gradient descent method. That is, we find the solution $t \mapsto(\Gamma(t), \boldsymbol{\rho}(t))$ of the following system of differential equations

$$
\begin{aligned}
\frac{\partial \phi}{\partial t} & =\left[\alpha \nabla_{\Gamma} E_{\text {Ray }}(\Gamma)+\gamma \nabla_{\Gamma} E_{\mathrm{I}}\left(\Gamma, T(\boldsymbol{\rho}) \Gamma_{0}\right)\right]|\nabla \phi|, \\
\dot{\boldsymbol{\rho}} & =-\beta \nabla_{\boldsymbol{\rho}} E_{\text {Ray }}\left(T(\boldsymbol{\rho}) \Gamma_{0}\right)-\gamma \nabla_{\boldsymbol{\rho}} E_{\mathrm{I}}\left(\Gamma, T(\boldsymbol{\rho}) \Gamma_{0}\right) .
\end{aligned}
$$

The $L^{2}$-shape gradients with respect to $\Gamma$ of the Rayleigh functional and the interaction term were computed in (6) and (8), respectively. The symbol $\nabla_{\rho}$ denotes the usual (finite dimensional) gradient w.r.t. the group parameters $\boldsymbol{\rho}$. The $\boldsymbol{\rho}$-derivatives are straight forward to compute and look similar to (but are not the same as) those computed in Yezzi et al. [11].

\section{Experiments}

We demonstrate our method in three experiments: one synthetic and two using ultrasound images. 


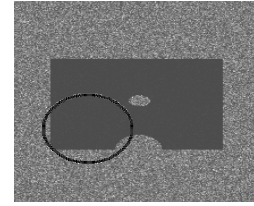

(a)

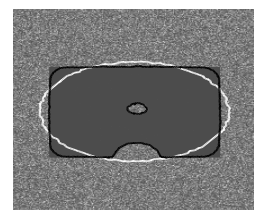

(d) SPS, $\gamma=0.24$

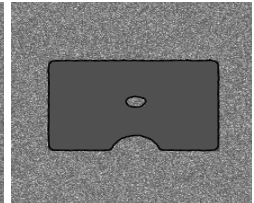

(b)

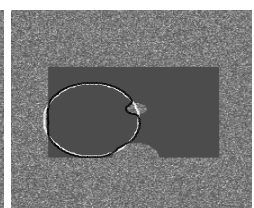

(e) SPS, $\gamma=0.25$

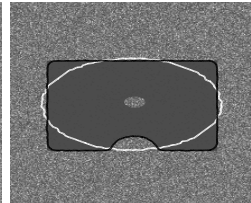

(c) $\mathrm{CAC}$

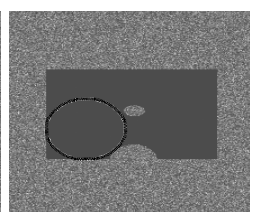

(f) SPS, $\gamma=1$
Figure 1: Synthetic experiment. Goal is to segment the structure without the oval inside. Prior (white) and active contour (black). (a) Initial position of prior and active contour, (b) Rayleigh segmentation without prior, $\approx 300$ its, (c) CAC, $\approx 250$ its, (d) SPS, $\approx 2200$ its, (e) SPS, $\approx 2000$ its, (f) $\mathrm{SPS}, \approx 500 \mathrm{its}$

The parameter $\gamma$ controls the interaction between the two contours. The active contour can move almost freely in a band of approximatively $W=\frac{1}{\sqrt[3]{\gamma}}$ pixels. Too large $\gamma$ will result in the two contours being very closely aligned and fixed in the image and too small $\gamma$ will result in the contours evolving independently of each other. The evolution is stopped when the active contour has remained stable for 20 iterations. We give only approximate $(\approx)$ number of iterations. The notation CAC = 'Coupled Active Contour' and SPS = 'Standard Prior Segmentation' is used in this section.

Synthetic Experiment. A synthetic image of intensity 100 is subjected to Rayleigh noise with $\sigma_{\text {int }}^{2}=1$ inside of a object (rectangle-like structure minus an oval disc) and $\sigma_{\text {ext }}^{2}=50$ in the background, cf. Figure 1 . We wish to segment the structure without the oval inside. This is sometimes relevant in medical applications, like endocardium segmentation, where we wish to segment only the endocardium and not structures within or close to the heart wall. Rayleigh segmentation without the use of a prior is ineffective. CAC can move the prior through the oval because it is sensitive to image data. The SPS approach is not equally effective; either the circle is not excluded from segmentation $(\gamma \leq 0.24)$ or the active contour becomes trapped by the oval $(\gamma>$ 0.24). Varying the curvature parameter $\nu$ did not improve the performance of SPS; it only moves the cut-off point (see (d) and (e)). CAC parameters: $\beta=100$, $\gamma=\left(\frac{1}{25}\right)^{3}$ i.e. $W=25$. Parameters for both methods: $\alpha=1, \nu=2$.
Segmentation of Echocardiograms. Before segmentation, graphics displaying the time of recording etc. are removed from the ultrasound image by applying a mask. The prior $\Gamma_{0}$ has a generic shape, roughly approximating the heart chamber. It was constructed by manually smoothing the mean shape of nine endocardium outlines from different patients at a specific moment in the cardiac cycle. In both experiments the transformation of the prior is affine, i.e. has 6 degrees of freedom. The curvature parameter $\nu$ is set high to ensure regular segmentations. Varying $\nu$ did not alter the results for SPS significantly, except the smoothness of the segmented contour.

US Experiment 1. For both SPS and CAC the active contour and prior are initialized in the center of the endocardium. In CAC segmentation the prior guides the active contour past the irrelevant dots in the chamber and ends up close to the outline given by clinician. Furthermore, the active contour is allowed to deviate from the prior, whereby segmentation of surrounding tissue is avoided. As is clearly seen in the top row of Figure 2 SPS cannot achieve a useful segmentation, since it cannot get past the irrelevant dots. If $\gamma$ is set low $(\approx 0.1)$ then the prior does not help the segmentation at all, and if set higher it still cannot help the active contour past the dots. This is due to the fact that the prior (in the standard method) does not interact with image. CAC parameters: $\beta=20, \gamma=\left(\frac{1}{3}\right)^{3}$ i.e. $W=3$. Parameters for both methods: $\alpha=1, \nu=7$.

US Experiment 2. We demonstrate the performance of CAC vs. SPS with respect to poor initialization. In Figure 3 we see that CAC converges nicely, while SPS fails. In SPS the prior is unable to guide the active contour to the chamber. For $\gamma<1$ SPS fails, since the active contour disappears. For $\gamma>1$ we obtain similar results as for $\gamma=1$. The ultrasound image used here is taken from a different part of the heart sequence than in Experiment 1. CAC parameters: $\beta=30, \gamma=\left(\frac{1}{3}\right)^{3}$ i.e. $W=3$. Parameters for both methods: $\alpha=1, \nu=15$.

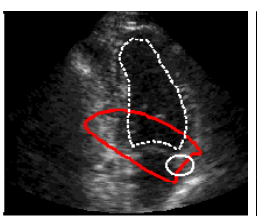

(a)

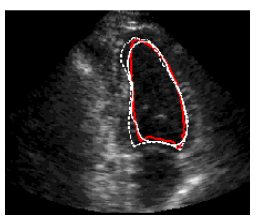

(b) $\mathrm{CAC}$

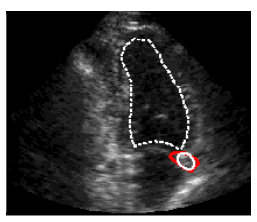

(c) SPS, $\gamma=1$
Figure 3: US Experiment 2: CAC vs. SPS w.r.t poor initialization. Clinician outline of heart chamber (dashed white), prior (red) and active contour (white). (a) Initialization, (b) $\mathrm{CAC}, \approx 500$ its, (c) SPS, $\approx 600$ its. 


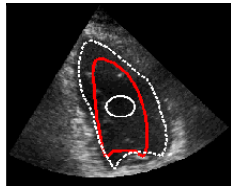

(a)

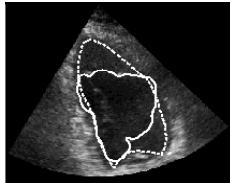

(b)

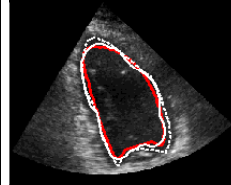

(c) $\mathrm{CAC}$

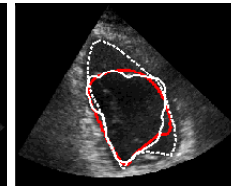

(d) SPS, $\gamma=0.1$

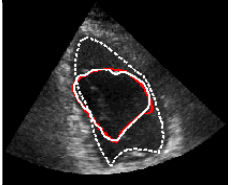

(e) SPS, $\gamma=0.5$

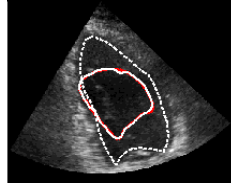

(f) SPS, $\gamma=0.75$

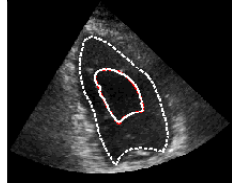

(g) SPS, $\gamma=1$

Figure 2: US Experiment 1: CAC vs. SPS with favorable initialization of active contour and prior. Clinician outline of heart chamber (dashed white), prior (red) and active contour (white). (a) Initialization, (b) Rayleigh segmentation without prior (c) CAC, $\approx 300$ its, (d) SPS, $\approx 2000$ its, (e) SPS, $\approx 1000$ its, (f) SPS, $\approx 1500$ its, (g) SPS, $\approx 2000$ its.
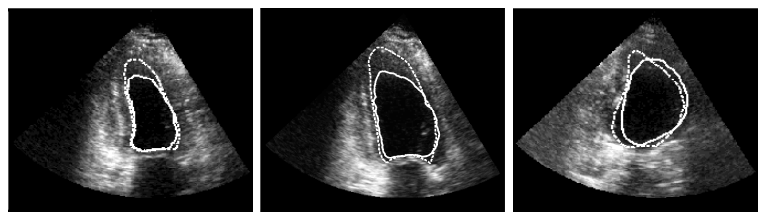

Figure 4: Rayleigh model in endocardium segmentation. Clinician outline of heart chamber (dashed white) and active contour (white). CAC segmentation of three different echocardiographic images. Note that upper part of endocardium is not segmented when using the Rayleigh model.

Rayleigh model in endocardium segmentation. We have observed that the Rayleigh statistics, used in both CAC and SPS model, does not sufficiently describe the endocardium. This is illustrated in Figure 4, where we have used CAC to segment the endocardium. It is clear that the upper part of the chamber is not modeled by the Rayleigh. This is a shortcoming of the statistical model, which influences the performance of CAC, since both active contour and sensitive prior depend on the Rayleigh model.

\section{Conclusions}

We have shown that the Coupled Active Contours (CAC) model, when applied to echocardiographic images, is more robust to image artifacts than a standard prior model, employing the area set symmetric difference measure for the interaction between prior and active contour. Furthermore we have observed that CAC is more tolerant to poor initialization and exhibits higher convergence rate. We have demonstrated the above through experiments where affine transformation of the prior is used in both real and synthetic cases.

\section{References}

[1] Y. Chen, F. Huang, H. D. Tagare, and M. Rao. A coupled minimization problem for medical image segmentation with priors. Int. Journal of Computer Vision, 71(3):259-272, 2007.

[2] Y. Chen, H. D. Tagare, S. Thiruvenkadam, F. Huang, D. Wilson, K. S. Gopinath, R. W. Briggs, and E. A. Geiser. Using Prior Shape in Geometric Active Contours in a Variational Framework. Int. Journal of Computer Vision, 50(3):315-328, 2002.

[3] D. Cremers, S. J. Osher, and S. Soatto. Kernel density estimation and intrinsic alignment for shape priors in level set segmentation. IJCV, 69(3):335-351, 2006.

[4] I. Dydenko, F. Jamal, O. Bernard, J. D'hooge, I. E. Magnin, and D. Friboulet. A level set framework with a shape and motion prior for segmentation and region tracking in echocardiography. Medical Image Analysis, 10(2):162-177, 2006.

[5] M. Leventon, W. Grimson, and O. Faugeras. Statistical shape influence in geodesic active contours. In $C V P R$, 2000.

[6] S. J. Osher and R. P. Fedkiw. Level Set Methods and Dynamic Implicit Surfaces. Springer Verlag, 2002.

[7] X. Qian, H. D. Tagare, and Z. Tao. Segmentation of Rat Cardiac Ultrasound Images with Large Dropout Regions. In Proc. CVPRW'06, 2006.

[8] T. Riklin-Raviv, N. Kiryati, and N. Sochen. Unlevelsets: Geometry and prior-based segmentation. In Computer Vision-ECCV 2004, volume 3024 of LNCS, pages 50-61. Springer, 2004.

[9] M. Rousson and N. Paragios. Shape priors for level set representations. In Proc. European Conf. on Computer Vision, volume 2351 of LNCS. Springer, 2002.

[10] A. Sarti, C. Corti, E. Mazzini, and C. Lamberti. Maximum Likelihood Segmentation of Ultrasound Images with Rayleigh Distribution. IEEE Trans. Ultrasonics, Ferroelectrics, and Frequency Control, 52(6):947-960, 2005.

[11] A. Yezzi, L. Zöllei, and T. Kapur. A variational framework for integrating segmentation and registration through active contours. Medical Image Analysis, 7(2):171-185, 2003. 